\title{
Peptide solids as a home for organic species and solid state reactions
}

\author{
Dmitriy V Soldatov, Aaron J Smith, Farukh I Ali \\ Department of Chemistry, University of Guelph, Guelph, ON, N1G 2W1, soldatov@uoguelph.ca
}

Short peptides often form crystalline solids with other molecules included as guests or crystal co-formers. The resulting inclusion compounds, co-crystals, or solids with an empty peptide framework resulted therefrom, appear to be sufficiently flexible to allow reversible sorption, guest exchange and solid state reactions.

Our research is focused on the design of crystals, co-crystals and inclusions with a predictable $\mathrm{H}$-bonded host matrix of peptide molecules. Short hydrophobic peptides frequently assembly into beta-sheet-like motifs with spacious interlayer gaps available to act as storage container or reaction vessel space. We found that several types of $\mathrm{H}$-bonded frameworks persistently form with a variety of peptides and template molecules, with parallel, antiparallel and crisscross arrangement of the molecules in the peptide layer. Once created, the frameworks are both stable and remarkably flexible, undergoing contraction/expansion or more sophisticated transformations without loosing their $\mathrm{H}$-bond connectivity. The properties of the new materials have been investigated. 